T93/079

\title{
$U(N)$ Gauge Theory and Lattice Strings
}

\author{
Ivan K. Kostov * \# \\ Service de Physique Théorique ${ }^{\dagger}$ de Saclay CE-Saclay, F-91191 Gif-Sur-Yvette, France
}

We explain, in a slightly modified form, an old construction allowing to reformulate the $U(N)$ gauge theory defined on a $D$-dimensional lattice as a theory of lattice strings (a statistical model of random surfaces). The world surface of the lattice string is allowed to have pointlike singularities (branch points) located not only at the sites of the lattice, but also on its links and plaquettes. The strings become noninteracting when $N \rightarrow \infty$. In this limit the statistical weight a world surface is given by $\exp [-$ area] times a product of local factors associated with the branch points. In $D=4$ dimensions the gauge theory has a nondeconfining first order phase transition dividing the weak and strong coupling phase. From the point of view of the string theory the weak coupling phase is expected to be characterized by spontaneous creation of "windows" on the world sheet of the string.

Talk delivered at the Workshop on string theory, gauge theory and quantum gravity, 28-29 April 1993, Trieste, Italy

August 1993

* on leave of absence from the Institute for Nuclear Research and Nuclear Energy, Boulevard Tsarigradsko Chaussée 72, BG-1784 Sofia, Bulgaria

\# (kostov@amoco.saclay.cea.fr)

$\dagger$ Laboratoire de la Direction des Sciences de la Matière du Comissariat à l'Energie Atomique 


\section{Introduction}

The concept of gluons as elementary excitations in QCD is adequate only at distances much less than the correlation length of the theory (the typical hadron radius). Because of the dimensional transmutation, the effective gauge coupling constant seizes to be a small parameter in the infrared domain and the standard perturbation theory collapses at distances comparable with the hadron radius.

An idea which is perhaps as old as QCD is that infrared-stable elementary excitations are described by fluctuating surfaces or strings. The Wilson's strong coupling expansion [1] and the $1 / N$ expansion of 't Hooft [2] are the two basic arguments in favour of the possibility of such a description.

From the point of view of the $1 / N$ expansion, the $U(N)$ gauge theory resembles a theory of strings with interaction constant $1 / N$ and its multicolor limit $N \rightarrow \infty$ is expected to be described by some kind of a noninteracting string. The formation of strings at large distances can be seen as a condensation of dense planar diagrams in the continuum theory (fig. 1). However, the only unambiguous evidence for the relevance of strings in the perturbation theory is the topology of the Feynman diagrams. Because of the divergencies and the need of gauge fixing, it is not easy to make this statement more rigorous.

Figure 1. A dence planar diagram for the Wilson loop average in the multicolour QCD

On the other hand, there is the strong coupling expansion in lattice gauge theories [1]. The gauge theory is discretized so that the gauge field is associated with the links $\ell=<x x^{\prime}>$ and the action is a sum over the elementary squares (plaquettes) of the lattice. From the point of view of the strong coupling expansion the lattice gauge theory is statistical mechanics of random surfaces with contact (self)interactions. In the lowest orders of the inverse coupling $\beta=1 / \lambda$, the Wilson loop average is given by the sum over lattice surfaces spanning the loop. In high orders, the dominant contribution comes from overlapping surfaces subjected to strong contact interactions and forming new topologies, and the original string picture is lost.

Some years ago, it has been suggested that the large $N$ limit of the strong coupling expansion might correspond to a theory of noninteracting surfaces. In order to study 
this possibility, the most commonly used character expansion was not the most efficient mathematical formalism because it produces diagrams for the partition function and not directly for the free energy. In spite of the considerable efforts [3] no evidence for a free string picture was obtained 1 .

An alternative formalism was then suggested by V. Kazakov [6]. The idea of [6], borrowed from the Stanley's solution [7] of the large- $N$ vector model, was to reformulate the $U(N)$ lattice gauge theory as a kind of Weingarten theory with additional interactions. The Weingarten model [8] is a simple (but rather pathological) gauge theory which is equivalent to a model of noninteracting random surfaces in the large $N$ limit. In the $U(N)$ lattice gauge theory one can impose the unitarity of the link variable $\mathbf{U}_{\ell}$ by means of a Lagrange multiplier and replace the Haar measure on the $U(N)$ group by a Gaussian measure over complex matrices. The idea of [6] has been accomplished in refs. [9], [10].

In ref. [11] we suggested the possibility that the strong coupling lattice surfaces and planar Feynman-'t Hooft diagrams appear as two extreme cases of the same perturbative expansion. This means that the strong coupling lattice string is perhaps not completely irrelevant to the continuum limit of QCD.

Here we explain the construction of ref. [11] using the standard formalism of the mean field analysis in gauge theories [12]. Our random surface Ansatz will be defined for any choice of the one-plaquette action. In $D=2$ dimensions it reproduces the continuum limit of the gauge theory in spite of eventual Gross-Witten transition [13] separating the strong and weak coupling domains. However, the first-order phase transition in $D=4$ dimensions is likely to persist and the sum over surfaces has to be modified in the weak coupling phase by allowing windows (free boundaries) to appear on the world sheet. In the continuum limit the world sheet of the string is tore into a network of thin strips which can be interpreted as gluon propagators.

We start the story with a review of the Stanley's solution [7] of the large- $N$ vector model which may help the reader to understand the logic of our construction before dipping into the technical details. The analogy with the vector model can help us to get some more intuition and avoid the rudest conceptual errors in the case of the gauge theory.

\section{The $U(N)$ vector model}

The fluctuating field in this model is an $N$-component complex vector $\mathbf{u}_{x}=$ $\left(u_{x}^{1}, \ldots, u_{x}^{N}\right)$ defined at the points $x$ of the $D$-dimensional hypercubical lattice and having unit norm

$$
\mathbf{u}_{x}^{*} \cdot \mathbf{u}_{x} \equiv \sum_{i=1}^{N}\left|u_{x}^{i}\right|^{2}=1
$$

1 Nevertheless, very interesting results concerning $\mathrm{QCD}_{2}$ have been obtained in a series of recent papers [4] using the method of characters. 
We assume a nearest neighbour interaction $\beta \mathbf{u}_{x}^{*} \cdot \mathbf{u}_{y}$ associated with the links $\ell=<x y>$ of the lattice. Then the measure

$$
[d \mathbf{u}]=\prod_{i=1}^{N} d \bar{u}^{i} d u^{i} \delta\left(\sum_{i=1}^{N}\left|u_{x}^{i}\right|^{2}-1\right)=d \mathbf{u}^{*} d \mathbf{u} \delta\left(\mathbf{u}^{*} \cdot \mathbf{u}-1\right)
$$

and the interaction are invariant under global $U(N)$ transformations

$$
\mathbf{u}_{x} \rightarrow \mathbf{U} \mathbf{u}_{x} ; \quad \mathbf{U} \in U(N)
$$

The partition function

$$
\mathcal{Z}=e^{N \mathcal{F}}=\int \prod_{x}\left[d \mathbf{u}_{x}\right] \prod_{\ell=<x y>} e^{N \beta \mathbf{u}_{x}^{*} \cdot \mathbf{u}_{y}}
$$

can be calculated order by order in the inverse coupling constant $\beta$ by expanding the exponential as a series of monomials (strong coupling expansion). This leads to a diagram technique which in the limit $N \rightarrow \infty$ involves tree-like clusters of loops and can be summed up explicitly in all orders.

Alternatively one can transform, by introducing auxiliary fields, the integral over the original vector field into a duable Gaussian integral and find an effective field theory in which the number $1 / N$ plays the rôle of a Planck constant.

Introducing a new complex vector field $\mathbf{h}=\left(h^{i}, i=1,2, \ldots, N\right)$ we represent the unimodular measure as a flat measure for the unconstrained complex vector field $\mathbf{v}=$ $\left(v^{i}, i=1, \ldots, N\right)$. We insert the exponential representation of the $\delta$-function

$$
\delta\left(u^{j}-v^{j}\right)=\frac{1}{2 \pi i} \int_{-i \infty}^{i \infty} d h_{j} e^{\bar{h}_{j}\left(u^{j}-v^{j}\right)}
$$

in the definition (2.4) of the partition function for every component $u_{x}^{j}$ of the vector field. Here $\mathbf{h}_{\ell}$ and $\mathbf{v}_{\ell}$ are unrestricted complex vectors on which the integration is performed with a flat measure

$$
d \mathbf{v}=\prod_{j=1}^{N} d v^{j} d \bar{v}^{j}, \quad d \mathbf{h}=\prod_{j=1}^{N} d h^{j} d \bar{h}^{j}
$$

Denoting by

$$
e^{N F\left(\mathbf{h}^{*} \cdot \mathbf{h}\right)}=\int[d \mathbf{u}] e^{N\left[\mathbf{u}^{*} \cdot \mathbf{h}+\mathbf{h}^{*} \cdot \mathbf{u}\right]}
$$

the one-site mean-field integral we write the partition function (2.4) in the form

$$
\mathcal{Z}=e^{N \mathcal{F}}=\int \prod_{x}\left(d \mathbf{h}_{x} d \mathbf{v}_{x} e^{-N\left(\mathbf{h}_{x}^{*} \cdot \mathbf{v}_{x}+\mathbf{h}_{x} \cdot \mathbf{v}_{x}^{*}\right)}\right) \prod_{x} e^{N F\left(\mathbf{h}_{x}\right)} \prod_{\ell=<x x^{\prime}>} e^{N \beta \mathbf{v}_{x} \cdot \mathbf{v}_{x}^{\prime}}
$$


The potential $F(\alpha)$ is determined by the Ward identity $\left(\sum_{i} N^{-2} \partial / \partial h^{i} \partial / \partial h_{i}^{*}-\right.$ 1) $e^{N F}=0$ or, in terms of the $\alpha$-field,

$$
\left(\frac{1}{N} \frac{\partial}{\partial \alpha}+\alpha \frac{1}{N^{2}} \frac{\partial^{2}}{\partial \alpha^{2}}-1\right) e^{N F(\alpha)}=0
$$

which is solved, in the large $N$ limit, by

$$
\begin{aligned}
\frac{\partial F}{\partial \alpha} & =\frac{\sqrt{1+4 \alpha}-1}{2 \alpha} \\
F(\alpha) & =\sqrt{1+4 \alpha}-1-\ln \frac{1+\sqrt{1+4 \alpha}}{2}=\sum_{n=1}^{\infty} f_{n} \frac{\alpha^{n}}{n}
\end{aligned}
$$

Using the integral representation

$$
e^{N F\left(\mathbf{h}^{*} \cdot \mathbf{h}\right)}=\int d \alpha d \tilde{\alpha} e^{N\left[\tilde{\alpha}\left(\mathbf{h}^{*} \cdot \mathbf{h}-\alpha\right)+F(\alpha)\right]}
$$

we finally write the partition function in a form allowing to perform exactly the integral over the vector fields

$$
\begin{aligned}
\mathcal{Z}=e^{N \mathcal{F}}= & \int \prod_{x} d \alpha_{x} d \tilde{\alpha}_{x} e^{N\left[F\left(\alpha_{x}\right)-\tilde{\alpha}_{x} \alpha_{x}\right]} d \mathbf{v}_{x} d \mathbf{h}_{x} e^{N\left[-\mathbf{v}_{x}^{*} \cdot \mathbf{h}_{x}-\mathbf{h}_{x}^{*} \cdot \mathbf{v}_{x}\right]} \\
& \prod_{x} e^{N\left[\tilde{\alpha}_{x} \mathbf{h}_{x}^{*} \cdot \mathbf{h}_{x}\right]} \prod_{\ell=<x x^{\prime}>} e^{N \beta \mathbf{v}_{x}^{*} \cdot \mathbf{v}_{x^{\prime}}}
\end{aligned}
$$

Denoting by $\hat{C}$ the connectivity matrix of the $D$-dimensional hypercubical lattice

$$
\hat{C}=\sum_{x} \sum_{\mu=1}^{D}\left(e^{\partial / \partial x_{\mu}}+e^{-\partial / \partial x_{\mu}}\right)
$$

we can write the result of the gaussian integration over the $\mathbf{v}, \mathbf{h}$ fields as

$$
e^{N \mathcal{F}}=\int \prod_{x} d \tilde{\alpha}_{x} d \alpha_{x} e^{N\left[F\left(\alpha_{x}\right)-\tilde{\alpha}_{x} \alpha_{x}\right]} e^{N \mathcal{F}_{0}[\tilde{\alpha}]} \equiv\left\langle e^{N \mathcal{F}_{0}[\tilde{\alpha}]}\right\rangle_{\tilde{\alpha}}
$$

where

$$
\mathcal{F}_{0}[\tilde{\alpha}]=-\sum_{x} \operatorname{tr} \log (1-\beta \tilde{\alpha} \hat{C})
$$

is the sum over vacuum loops of the vector fields.

Formally the sum over vacuum loops can be obtained by expanding the exponential in the last line of (2.12) as a sum of monomials and applying all possible Wick contractions

$$
\left\langle\mathbf{h}_{x}^{* i} \mathbf{v}_{y j}\right\rangle=\delta_{x, y} \delta_{j}^{i},\left\langle\mathbf{v}_{x}^{* i} \mathbf{h}_{y j}\right\rangle=\delta_{x, y} \delta_{j}^{i}
$$


The vertex $\tilde{\alpha}_{x} \mathbf{h}_{x}^{*} \cdot \mathbf{h}_{x}$ is represented by a node at the point $x$ and contributes a factor $\tilde{\alpha}_{x}$ to the weight of the loop. The vertex $\beta \mathbf{v}_{x}^{*} \mathbf{v}_{y}$ is represented by a segment of the loop covering the link $\ell=\langle x y>$ and contributes a factor $\beta$. The weight of each loop is therefore a product of local factors $\beta$ and $\tilde{\alpha}_{x}$ associated with its nodes and links, correspondingly. Denoting by $\Gamma$ any closed loop on the lattice, the r.h.s. of (2.15) can be written as

$$
\mathcal{F}_{0}\left[h^{*}\right]=\sum_{\Gamma} \beta^{\# \text { links }} \prod_{x \in \Gamma} \tilde{\alpha}_{x}
$$

The action for the fields $\tilde{\alpha}, \alpha$ is proportional to $N$ and in the limit $N \rightarrow \infty$ these fields freeze at their expectation values determined by the classical equations of motion

$$
\begin{gathered}
\tilde{\alpha}=\frac{\partial F(\alpha)}{\partial \alpha}=\frac{\sqrt{1+4 \alpha}-1}{2 \alpha} \\
\alpha=\frac{\partial \mathcal{F}_{0}(\tilde{\alpha})}{\partial \tilde{\alpha}}=\frac{\beta \hat{C}}{1-\tilde{\alpha} \beta \hat{C}}
\end{gathered}
$$

or, equivalently,

$$
\tilde{\alpha}+\alpha \tilde{\alpha}^{2}=1, \quad \frac{\tilde{\alpha}}{1-\beta \tilde{\alpha} \hat{C}}=1
$$

The last equation has a simple geometrical interpretation in terms of a random walk on the lattice. It can be obtained also directly from the unimodularity condition $\mathbf{u}_{x}^{*} \cdot \mathbf{u}_{x}=1$. Consider the two-point Greens function $G_{x y}=\left\langle\mathbf{u}_{x}^{*} \cdot \mathbf{u}_{y}\right\rangle$. Repeating the same steps as for the partition function we find

$$
G_{x y}=\left\langle\mathbf{v}_{x}^{*} \cdot \mathbf{v}_{y}\right\rangle=\left\langle x\left|\frac{\tilde{\alpha}}{1-\beta \hat{C}}\right| y\right\rangle=\sum_{\Gamma_{x y}} \tilde{\alpha}^{\# \text { nodes }} \beta^{\# \text { links }}
$$

Therefore the second of the equations (2.20) is just the condition

$$
G_{x x}=1 .
$$

We see that the original vector field $\mathbf{u}_{x}$ acquires a longitudinal degree of freedom and instead of $2 N-1$ we have $2 N$ degrees of freedom at each point $x$. These describe $N$ identical noninteracting particles with dynamically generated mass. The constraint $\mathbf{u}_{x}^{*} \cdot \mathbf{u}_{x}=1$ is imposed on dynamical level by the condition (2.22) which determines the mass gap of the model. After diagonalysing the connectivity matrix we write it as an integral in the momentum space

$$
\begin{gathered}
\int_{-\pi}^{\pi} \frac{1}{m^{2}+\sum_{\mu=1}^{D}\left[2 \sin \left(p_{\mu} / 2\right)\right]^{2}}=\beta \\
m^{2}=\frac{1}{\tilde{\alpha} \beta}-2 D
\end{gathered}
$$

The continuum limit (infinite correlation length) is achieved when $m^{2} \rightarrow 0$, i.e., $2 D \beta \tilde{\alpha}=1$.

Let us examine more closely this equation depending on the dimension $D$. 


\section{1. $D \leq 2$}

When $D \leq 2$, the integral is infrared divergent when $m \rightarrow 0$ and gives

$$
\beta=\int \frac{d^{D} p}{(2 \pi)^{D}} \frac{1}{p^{2}+m^{2}}=m^{D-2}, \quad D<2
$$

In the marginal case $D=2$ we find the asymptotic freedom law for the dependence of the renormalized coupling $\beta=\beta(m)$ as a function of the correlation length $1 / m$

$$
\beta=\frac{1}{4 \pi} \ln \frac{1}{m^{2}}
$$

\section{2. $D>2$}

Above two dimensions the integral is convergent at $m \rightarrow 0$ and the limit of zero mass is achieved at finite value of $\beta=\beta_{c}$.

In the weak coupling phase $\beta>\beta_{c}$ the equations of motion (2.23) have no solution which means that something in our construction is wrong. This contradiction is resolved if we admit that the vector fields acquire nonzero vacuum expectation values $\left\langle\mathbf{v}_{x}\right\rangle=\mathbf{v}_{\mathrm{cl}}$, $\left\langle\mathbf{h}_{x}\right\rangle=\mathbf{h}_{\mathrm{cl}}$ determined by the saddle-point equations

$$
\mathbf{h}_{\mathrm{cl}}=\beta \hat{C} \mathbf{v}_{\mathrm{cl}}=2 D \beta \mathbf{v}_{\mathrm{cl}}, \quad \mathbf{v}_{\mathrm{cl}}=\tilde{\alpha} \mathbf{h}_{\mathrm{cl}}
$$

which are solved by $\mathbf{h}_{\mathrm{cl}}=\mathbf{v}_{\mathrm{cl}}=0$ ( strong coupling $U(N)$-symmetric phase) or by $m^{2}=$ $1-2 D \beta \tilde{\alpha}=0$ with $\mathbf{h}_{\mathrm{cl}}, \mathbf{v}_{\mathrm{cl}} \neq 0$ ( weak coupling phase with spontaneously broken $U(N)$ symmetry). Geometrically the weak coupling phase is characterized by possibility of the the random walk to break into two pieces. Then the two-point Greens function is a sum of connected and disconnected parts

$$
G_{x y}=\mathbf{v}_{\mathrm{cl}}^{2}+\left\langle x\left|\frac{\tilde{\alpha}}{1-\tilde{\alpha} \beta \hat{C}}\right| y\right\rangle
$$

The unimodularity condition now reads

$$
\mathbf{v}_{\mathrm{cl}}^{2}+\frac{1}{\beta} \int \frac{d^{D} p}{(2 \pi)^{D}} \frac{1}{\sum_{\mu=1}^{D}\left(2 \sin \left(p_{\mu} / 2\right)\right)^{2}}=\mathbf{v}_{\mathrm{cl}}^{2}+\frac{\beta_{c}}{\beta}=1
$$

Thus in the weak coupling phase there is a spontaneous magnetization $\mathbf{v}_{\mathrm{cl}}=\sqrt{1-\beta_{c} / \beta}<$ 1 and $2 N-1$ Goldstone excitations with zero mass due to the spontaneous breaking of the $U(N)$ symmetry.

The critical value $\beta_{c}$ is related to the probability $\Pi_{0}$ that a Brownian walk returns to the origin 14

$$
2 D \beta_{c}=\frac{1}{1-\Pi_{0}}
$$


and its large behaviour is given by

$$
2 D \beta_{c}=1+\frac{1}{2 D}+3\left(\frac{1}{2 d}\right)^{2}+\ldots
$$

The series diverges at $D=2$ and as $D \rightarrow 2, \beta_{c} \rightarrow \infty$

$$
2 D \beta_{c} \sim \frac{2}{\pi(D-2)}
$$

and below the lower critical dimension $D=2$ no transition occurs. In $D<2$ there exists only a high temperature symmetric phase.

\section{3. $D=1$}

The $D=1$ vector model has no dynamical degrees of freedom and is analogous to the $D=2$ gauge theory.

In terms of the weak coupling parameter $\lambda$ related to $\beta$ by $2 / \beta=\sinh (\lambda / 2)$ we find

$$
\tilde{\alpha}=\tanh \frac{\lambda}{2}, \quad \beta=\frac{1}{2 \sinh \lambda / 2}
$$

and the two-point correlator is given by

$$
G_{x y}=e^{-(\lambda / 2)|x-y|}
$$

The two-point correlator can be interpreted either as the sum over all random walks (with backtrackings) connecting the points $x$ and $y$, or as the only minimal path (without backtrackings) connecting the two points, with a weight factor $G_{1}=e^{-\lambda / 2}$ associated with each of its links. In the first interpretation the mean value of $\tilde{\alpha}$ is tuned so that it compensates completely the entropy due to backtrackings. In the case of the gauge theory the analog of the backtrackings are the folds of the world sheet of the string. We shall see that the $D=2$ gauge theory can be formulated in terms of minimal nonfolding surfaces.

\section{The $U(N)$ gauge theory}

In the Wilson-Polyakov formulation of the $U(N)$ lattice theory [1] the independent fluctuating variable is a unitary matrix $\mathbf{U}_{\ell}=\left\{\left(U_{\ell}\right)_{i}^{j}, i, j=1, \ldots, N\right\}$

$$
\mathbf{U}_{\ell}^{\dagger} \mathbf{U}_{\ell}=\mathbf{I}
$$


associated with the oriented links $\ell=\langle x y>$ of the hypercubical lattice 2 . The measure and the interaction are invariant with respect to local gauge transformations

$$
\mathbf{U}_{<x y>} \rightarrow \mathbf{U}_{x} \mathbf{U}_{<x y>} \mathbf{U}_{y}^{\dagger}
$$

The amplitude $\mathbf{U}(\Gamma)$ associated with the parallel transport along the loop $\Gamma=\left\{\ell_{1} \ell_{2} \ldots \ell_{n}\right\}$ is given by the product of the link variables

$$
\mathbf{U}(\Gamma)=\mathbf{U}_{\ell_{1}} \mathbf{U}_{\ell_{2}} \ldots \mathbf{U}_{\ell_{n}}
$$

The action in the gauge theory is a functional on the loop fields (3.3). The simplest nontrivial loop $\Gamma$ is the boundary $\partial p$ of a plaquette $p$, an elementary square on the hypercubical lattice. In what follows we will denote by $\mathbf{U}_{p}$ the corresponding loop variable, the ordered product of the 4 link variables along the boundary of the plaquette $p$. Let us denote by $S_{\lambda}(\mathbf{U})$ the one-plaquette action where $\lambda$ is the coupling constant of the lattice gauge theory. Then the partition function is defined by the integral over all link variables

$$
Z=e^{N^{2} \mathcal{F}}=\int_{\ell \in \mathcal{S}}\left[d \mathbf{U}_{\ell}\right] \prod_{p} e^{S_{\lambda}\left(\mathbf{U}_{p}\right)}
$$

where

$$
[d \mathbf{U}]=d \mathbf{U} d \mathbf{U}^{\dagger} \delta\left(\mathbf{U}^{\dagger} \mathbf{U}-\mathbf{I}\right) \equiv \prod_{i, j=1}^{N} d U_{i}^{j} d\left(U^{\dagger}\right)_{i}^{j} \delta\left(\sum_{k}\left(U^{\dagger}\right)_{i}^{k} U_{k}^{j}-\delta_{i}^{j}\right)
$$

is the invariant measure on the group $U(N)$. By $\mathcal{F}$ we denoted the free energy per degree of freedom.

The one-plaquette action is subjected to the following two requirements. First, it should be a real function defined on the conjugacy classes of the group $U(N)$. This means that it can be expanded in the characters of the irreducible representations of the group $U(N)$

$$
e^{N^{2} S_{\lambda}(\mathbf{U})}=\sum_{-\infty \leq n_{1} \leq \ldots \leq n_{N} \leq \infty} \chi_{\vec{n}}(\mathbf{I}) \chi_{\vec{n}}(\mathbf{U}) e^{N^{2} S_{\lambda}^{\vec{n}}}
$$

The character of the representation with signature $\vec{n}=\left\{n_{1}, \ldots, n_{N}\right\}$ depends on the eigenvalues $u_{1}, \ldots, u_{N}$ of the unitary matrix $\mathbf{U}$ as

$$
\chi_{\vec{n}}(\mathbf{U})=\frac{\operatorname{det}_{i k}\left(u_{i}^{n_{k}+k-1}\right)}{\operatorname{det}_{i k}\left(u_{i}^{k-1}\right)} .
$$

2 We will use the notation $\ell^{-1}=<y x>$ for the link obtained from $\ell=\langle x y>$ by reversing the orientation. More generally, if $\Gamma_{1}$ and $\Gamma_{2}$ are two oriented lines, the product $\Gamma_{1} \Gamma_{2}$ is defined, in case it exists, as the line obtained by identifying the end of $\Gamma_{1}$ with the beginning of $\Gamma_{2}$. 
Second, we assume that the continuum limit is achieved when $\lambda \rightarrow 0$ and in this limit

$$
S_{\lambda}(\mathbf{U}) \rightarrow-\frac{1}{2} \frac{\operatorname{tr}}{N} \mathbf{A}^{2}, \quad \mathbf{U}=e^{i \sqrt{\lambda} \mathbf{A}}
$$

The Fourier image of the action in this limit is proportional to the second Cazimir operator

$$
S_{\lambda}^{\vec{n}}=\frac{\lambda}{2 N} C_{2}(R)=\frac{\lambda}{2 N}\left(\sum_{i=1}^{N} n_{i}^{2}+\sum_{i<j}\left(n_{i}-n_{j}\right)\right)
$$

If we assume that eq. (3.9) remains true also for finite values of $\lambda$, the corresponding one-plaquette action is known as heat kernel action [15], $S_{\lambda}^{H K}(\mathbf{U})$. The exponential of this action is a solution of the heat kernel equation on the group manifold

$$
\left[2 \frac{\partial}{\partial \lambda}+(\mathbf{U} \partial / \partial \mathbf{U})^{2}\right] e^{N^{2} S_{\lambda}^{H K}(\mathbf{U})}=0
$$

The heat kernel action has the nice property of reproducing itself after group integration 16]

$$
\int[d \mathbf{U}] e^{N^{2} S_{\lambda_{1}}^{H K}\left(\mathbf{U}_{1} \mathbf{U}\right)} e^{N^{2} S_{\lambda_{2}}^{H K}\left(\mathbf{U}_{2} \mathbf{U}^{-1}\right)}=e^{N^{2} S_{\lambda_{1}+\lambda_{2}}^{H K}\left(\mathbf{U}_{1} \mathbf{U}_{2}\right)}
$$

Another simple choice is the Wilson action [1] $S_{\lambda}^{\mathrm{Wils}}(\mathbf{U})=\frac{1}{2 \lambda} \frac{\operatorname{tr}}{N}\left(\mathbf{U}+\mathbf{U}^{\dagger}\right)$. Its Fourier image is given by two different analytic expressions in the limit $N \rightarrow \infty$, depending on the value of the coupling constant. For example, for the fundamental representation $\vec{n}_{f}=[0,0, \ldots, 0,1]$

$$
S_{\lambda}^{\vec{n}_{f}}= \begin{cases}\frac{1}{2 \lambda}, & \text { if } \lambda>1 \\ 1-\frac{1}{2} \lambda, & \text { if } \lambda<1\end{cases}
$$

The nonanalyticity at the point $\lambda=1$ known as Gross-Witten phase transition [13] is due to the fact that the integral for the inverse Fourier transform

$$
e^{N^{2} S_{\lambda}^{\vec{n}}}=\frac{1}{\chi_{\vec{n}}(\mathbf{I})} \int[d \mathbf{U}] e^{N^{2} S_{\lambda}(\mathbf{U})} \chi_{\vec{n}}(\mathbf{U})
$$

is saturated by the vicinity of a saddle point and the saddle-point solution can have two analytic branches. In the strong coupling domain $\lambda>1$ the eigenvalues of the unitary matrix $\mathbf{U}$ are distributed all along the unit circle, and in the weak coupling domain $\lambda<1$ the density of the phases of the eigenvalues is supported by an interval $[-\phi, \phi]$ with $\phi<\pi$. 


\subsection{The gauge theory in terms of nonrestricted complex matrices}

Now let us convert this model into a theory with flat measure and regular interaction using the Laplace transform of the measure in the fields as in the $U(N)$ vector model. This has been actually done in the mean field analysis of lattice gauge theories [12]. We insert the exponential representation of the $\delta$-function

$$
\delta\left(U_{i}^{j}-V_{i}^{j}\right)=\frac{1}{2 \pi i} \int_{-i \infty}^{i \infty} d H_{j}^{i} e^{\bar{H}_{j}^{i}\left(U_{i}^{j}-V_{i}^{j}\right)}
$$

in the definition of the partition function for every matrix element $\left(U_{\ell}\right)_{i}^{j}$ of the gauge fields. Here $\mathbf{V}_{\ell}$ and $\mathbf{H}_{\ell}$ are unrestricted complex matrices on which the integration is performed with a flat measure

$$
d \mathbf{V}=\prod_{i, j=1}^{N} d V_{j}^{i} d \bar{V}_{j}^{i}, d \mathbf{H}=\prod_{i, j=1}^{N} d H_{j}^{i} d \bar{H}_{j}^{i}
$$

For each oriented link $\ell=<x y>$ only one pair of these fields is introduced and the convention

$$
\mathbf{V}_{<y x>}=\mathbf{V}_{\ell^{-1}}=\mathbf{V}_{\ell}^{\dagger}=\mathbf{V}_{<x y>}^{\dagger}, \quad \mathbf{H}_{<y x>}=\mathbf{H}_{\ell^{-1}}=\mathbf{H}_{\ell}^{\dagger}=\mathbf{H}_{<x y>}^{\dagger}
$$

is assumed. Denoting by

$$
e^{N^{2} F(\mathbf{H})}=\int[d \mathbf{U}] e^{N \operatorname{tr}\left(\mathbf{H}^{\dagger} \mathbf{U}+\mathbf{H} \mathbf{U}^{\dagger}\right)}
$$

the one-link mean-field integral we write the partition function (3.4) in the form

$$
\begin{aligned}
\mathcal{Z}=e^{N^{2} \mathcal{F}} & =\int \prod_{\ell}\left(d \mathbf{H}_{\ell} d \mathbf{V}_{\ell} e^{-N \operatorname{tr}\left(\mathbf{H}_{\ell}^{\dagger} \mathbf{V}_{\ell}+\mathbf{H}_{\ell} \mathbf{V}_{\ell}^{\dagger}\right)}\right) \\
& \prod_{\ell} e^{N^{2} F\left(\mathbf{H}_{\ell}\right)} \prod_{p} e^{N^{2} S\left(\mathbf{V}_{p}\right)}
\end{aligned}
$$

where $\mathbf{V}_{p}$ denotes the ordered product of link variables along the boundary $\partial p$ of the elementary square $p$

$$
\mathbf{V}_{p}=\prod_{\ell \in \partial p} \mathbf{V}_{\ell}
$$

The measure is now flat and the integrand is regular. The constraint (3.1) is satisfied in the sense of mean values

$$
\left\langle\left(\mathbf{V}_{\ell}^{\dagger} \mathbf{V}_{\ell}-\mathbf{I}\right) \ldots\right\rangle=0
$$

In order to give topological meaning of the $1 / N$ expansion we need to express the two potentials $F$ and $S_{\lambda}$ in terms of the moments $\operatorname{tr}(\cdot)^{n}, n=1,2, \ldots$, of their arguments. 


\subsection{The potential for the $\mathbf{H}$-field}

The function $F$ can be determined using the Ward identity

$$
\left[\operatorname{tr}\left(\partial / \partial \mathbf{H}^{\dagger} \partial / \partial \mathbf{H}\right)-N\right] e^{F\left(\mathbf{H}^{\dagger} \mathbf{H}\right)}=0
$$

which is the matrix analogue of (2.9). In the large- $N$ limit this function was calculated explicitly by Brezin and Gross [17 in terms of the eigenvalues of the Hermitean matrix $\mathbf{H}^{\dagger} \mathbf{H}$. For sufficiently small field $\mathbf{H}$ it can be expanded as a series in the momenta

$$
\begin{gathered}
\alpha_{n}=\frac{\operatorname{tr}}{N}\left(\mathbf{H}^{\dagger} \mathbf{H}\right)^{n}, \\
F[\alpha]=\sum_{n=1}^{\infty} \sum_{k_{1}, \ldots, k_{n} \geq 1} f_{\left[k_{1}, \ldots, k_{n}\right]} \frac{\alpha_{k_{1}} \ldots \alpha_{k_{n}}}{n !}
\end{gathered}
$$

The coefficients of the series were found in the large $N$ limit by O'Brien and Zuber [18] as a solution of a system of algebraic relations suggested originally by Kazakov in [6]. These relations are equivalent to eq. (3.21) written in terms of loop variables

$$
\left(\sum_{n \geq 1} n \alpha_{n-1} \frac{\partial_{n}}{N^{2}}+\sum_{k, n \geq 1}\left[(n+k+1) \alpha_{n} \alpha_{k} \frac{\partial_{k+n+1}}{N^{2}}+n k \alpha_{n+k-1} \frac{\partial_{n}}{N^{2}} \frac{\partial_{k}}{N^{2}}\right]-1\right) e^{N^{2} F[\alpha]}=0
$$

where we denoted $\alpha_{0}=1, \partial_{n}=\partial / \partial \alpha_{n}$. Eq. (3.24) is sufficient to determine the $1 / N$ expansion of the link-vertices $f_{\left[k_{1}, \ldots, k_{n}\right]}$. In the large $N$ limit the identity (3.24) is equivalent to a system of recurrence relations [9]

$$
\begin{aligned}
f_{[1]} & =1 \\
f_{\left[k_{1}, \ldots, k_{n}\right]}+\sum_{j=2}^{n} k_{j} f_{\left[k_{1}+k_{j}, k_{2}, \ldots, \bar{k}_{j}, \ldots, k_{n}\right]}+\sum_{k=1}^{k_{1}-1} \sum_{\alpha \in P(L)} f_{\left[k_{1}-k, L \backslash \alpha\right]} f_{[k, \alpha]} & =0
\end{aligned}
$$

where $L=k_{2}, \ldots, k_{n}$ and $P(L)$ is the set of all subsets of $L$, including the emply set, and the bar means omitting the argument below it. The equations for the weights $f_{[k]}$ of the disks [6]

$$
f_{[1]}=1 ; \quad f_{[n]}+\sum_{k=1}^{n-1} f_{[k]} f_{[n-k]}=0, n=1,2, \ldots
$$

are solved by the Catalan numbers

$$
f_{[n]}=(-)^{(n-1)} \frac{(2 n-2) !}{n !(n-1) !}
$$




\subsection{The potential for the $\mathbf{V}$ field}

The potential $S_{\lambda}(V)$ is defined by the analytic continuation of the r.h.s. of (3.6) from the hyperplane $\mathbf{V}=\mathbf{U}, \quad \mathbf{U}^{\dagger} \mathbf{U}=\mathbf{I}$ to the space of all complex matrices $\mathbf{V}$.

Using the explicit formula for the characters (3.7) we can express the r.h.s. of (3.6) as a series in the moments

$$
\beta_{n}=\frac{\operatorname{tr}}{N} \mathbf{V}^{n}, \beta_{-n}=\frac{\operatorname{tr}}{N} \mathbf{V}^{\dagger n} ; \quad n=1,2, \ldots
$$

The series is convergent for small $\mathbf{V}$ and can be represented as exponential of another series

$$
N^{2} S_{\lambda}[\beta]=N^{2} \sum_{n=1}^{\infty} \sum_{k_{1}, \ldots, k_{n} \neq 0} s_{\left[k_{1} \ldots k_{n}\right]} \frac{\beta_{k_{1} \ldots \beta_{k_{n}}}}{n !}
$$

In the continuum limit $\lambda \rightarrow 0$ the expansion (3.29) takes the form B $^{2}$

$$
S_{0}(\mathbf{V})=\sum_{n=1}^{\infty} \frac{1}{n}\left(\beta_{n}+\beta_{-n}-\beta_{n} \beta_{-n}\right)=\prod_{i, j-1}^{N} \frac{1-v_{i} \bar{v}_{j}}{\left(1-v_{i}\right)^{N}\left(1-\bar{v}_{j}\right)^{N}}
$$

which tends to the $\delta$-function $\delta(\mathbf{V}, \mathbf{I})$ when $\mathbf{V}$ approaches the hypersurface $\mathbf{V}^{\dagger} \mathbf{V}=\mathbf{I}$.

Note that the potential (3.29) for the Wilson action has the form $\operatorname{tr}\left(\mathbf{V}+\mathbf{V}^{\dagger}\right)$ only in the strong coupling domain. In the weak coupling domain $\lambda<1$ it is given by the most general expansion (3.29) with complicated coefficients. Therefore the heat kernel action is simpler to work with in the weak coupling regime.

The lowest-order coefficients for the heat kernel action are, in the large $N$ limit

$$
\begin{aligned}
& s_{[1]}=s_{[-1]}=e^{-\lambda / 2}, s_{[2]}=s_{[-2]}(1-\lambda) e^{-\lambda}, \\
& s_{[1,1]}=s_{[-1,-1]}=\left(\lambda-\lambda^{2} / 2\right) e^{-\lambda}, s_{[1,-1]}=-e^{-\lambda}
\end{aligned}
$$

\subsection{The $U(N)$ gauge theory à la Weingarten}

The interpretation of the functional integral as a sum over surfaces is possible if the action is linear in the traces of the matrix fields. This is achieved by the integral transformation

$$
e^{N^{2} F\left(\mathbf{H}^{\dagger} \mathbf{H}\right)}=\int \prod_{n=1}^{\infty}\left(d \alpha_{n} d \tilde{\alpha}_{n} e^{N^{2}\left[\frac{\tilde{\alpha}_{n}}{n} \frac{\operatorname{tr}}{N}\left(\mathbf{H}^{\dagger} \mathbf{H}\right)^{n}-\frac{\tilde{\alpha}_{n} \alpha_{n}}{n}\right]}\right) e^{N^{2} F[\alpha]}
$$

3 This formula resembles eq. (22) in the paper by M. Douglas [19. We tried to establish an exact correspondence between his formalism and ours, but haven't succeed in this 
By means of another system of parameters we represent the exponential of the plaquette action in the form

$$
e^{N^{2} S(\mathbf{V})}=\int \prod_{n=1}^{\infty}\left(d \beta_{n} d \tilde{\beta}_{n} d \beta_{-n} d \tilde{\beta}_{-n} e^{N^{2}\left[\frac{\tilde{\beta}_{n}}{n}\left(\frac{\operatorname{tr}}{N} \mathbf{V}^{n}-\beta_{n}\right)+\frac{\tilde{\beta}_{-n}}{n}\left(\operatorname{tr} \mathbf{V}^{\dagger n}-\beta_{-n}\right)\right]}\right) e^{N^{2} S[\beta]}
$$

We therefore introduce at each link $\ell$ and at each elementary square $p$ a set of auxiliary loop variables coupled to the moments of the matrix fields

$$
\begin{gathered}
\alpha_{n}(\ell), \tilde{\alpha}_{n}(\ell) ; \quad n=1,2, \ldots \\
\beta_{n}(p), \tilde{\beta}_{n}(p) ; \quad n= \pm 1, \pm 2, \ldots
\end{gathered}
$$

and represent the integral (3.18) as a theory of Weingarten type described by the partition function

$$
\begin{aligned}
\mathcal{Z}=e^{N^{2} \mathcal{F}} & =\left\langle\int \prod_{\ell} d \mathbf{H}_{\ell} d \mathbf{V}_{\ell} e^{-N \operatorname{tr}\left(\mathbf{H}_{\ell}^{\dagger} \mathbf{V}_{\ell}+\mathbf{H}_{\ell} \mathbf{V}_{\ell}^{\dagger}\right)}\right. \\
& \left.\prod_{\ell ; n>0} e^{\frac{N}{n} \tilde{\alpha}_{n}(\ell) \operatorname{tr}\left(\mathbf{H}_{\ell}^{\dagger} \mathbf{H}_{\ell}\right)^{n}} \prod_{p ; n>0} e^{\frac{N}{n}\left[\tilde{\beta}_{n}(p) \operatorname{tr} \mathbf{V}_{p}^{n}+\tilde{\beta}_{-n} \operatorname{tr} \mathbf{V}_{p}^{\dagger}\right]}\right\rangle_{\tilde{\alpha}, \tilde{\beta}}
\end{aligned}
$$

where the Boltzmann weights of the surfaces are themselves quantum fields and the average with respect to them is defined by

$$
\begin{aligned}
\langle *\rangle_{\tilde{\alpha}, \tilde{\beta}} & =\int \prod_{\ell \in \mathcal{L} ; n>0} d \alpha_{n}(\ell) d \alpha_{n}(\ell) e^{-N^{2} \frac{\tilde{\alpha}_{n}(\ell) \alpha_{n}(\ell)}{n}+F[\alpha(\ell)]} \\
& \prod_{p ; n \neq 0} d \tilde{\beta}_{n}(p) d \beta_{n}(p) e^{-N^{2} \frac{\tilde{\beta}_{n}(p) \beta_{n}(p)}{n}+S[\beta(p)]_{*}}
\end{aligned}
$$

The integration over the matrix fields in (3.36) will produce an effective action which will be interpreted in the next section as a sum over closed connected surfaces on the lattice. The leading term in this action is proportional to $N^{2}$. Therefore, in the limit $N \rightarrow \infty$ the integral over the scalar fields (3.34) and (3.35) is saturated by a saddle point and these fields freeze at their vacuum expectation values

$$
\left\langle\tilde{\alpha}_{n}(\ell)\right\rangle=\tilde{\alpha}_{n}, \quad\left\langle\tilde{\beta}_{n}(p)\right\rangle=\left\langle\tilde{\beta}_{-n}(p)\right\rangle=\tilde{\beta}_{n}
$$

This is natural to expect since the auxiliary scalar fields are coupled to gauge invariant collective excitations of the original matrix fields (the moments $(\cdot)^{n}$ ) which, due to selfaveraging, become classical in the large $N$ limit. The classical values of the fields $\tilde{\alpha}_{n}$ can be determined from the stationarity condition for the effective action, or, alternatively, from 
the Ward identity (3.20). Thus in the limit $N \rightarrow \infty$ we obtain a theory of noninteracting planar $(G=0)$ random surfaces with Boltzmann weights determined dynamically. This picture is conceptually the same as in the $U(N)$ vector model.

Since we are interested only in the large $N$ limit, the fields (3.34) and (3.35) can be replaced with their vacuum expectation values even before the integration over the matrix fields. We therefore find the following expression for the free energy of the $U(\infty)$ gauge theory

$$
\begin{aligned}
\mathcal{F}=\lim _{N \rightarrow \infty} \frac{1}{N^{2}} \log ( & \int \prod_{\ell} d \mathbf{H}_{\ell} d \mathbf{V}_{\ell} e^{-N \operatorname{tr}\left(\mathbf{H}_{\ell}^{\dagger} \mathbf{V}_{\ell}+\mathbf{H}_{\ell} \mathbf{V}_{\ell}^{\dagger}\right)} \\
& \left.\prod_{\ell ; n>0} e^{\frac{N}{n} \tilde{\alpha}_{n} \operatorname{tr}\left(\mathbf{H}_{\ell}^{\dagger} \mathbf{H}_{\ell}\right)^{n}} \prod_{p ; n>0} e^{\frac{N}{n} \tilde{\beta}_{n}\left(\operatorname{tr} \mathbf{V}_{p}^{n}+\operatorname{tr} \mathbf{V}_{p}^{\dagger n}\right)}\right)
\end{aligned}
$$

The values $\alpha_{n}, \tilde{\beta}_{n}$ should be kept as free parameters. The parameters $\tilde{\alpha}_{n}$ are determined by the condition of unitarity $(3.20)$ and $\tilde{\beta}_{n}$ can be considered as coupling constants defining the plaquette action.

\section{Branched random surfaces}

\subsection{Feynman rules in the large $N$ limit}

Assuming that the saddle point for the integral (3.18) is the trivial field $\mathbf{H}=\mathbf{V}=0$, we can express the free energy as a sum of vacuum Feynman diagrams.

The Feynman rules are obtained as follows. Inverting the bilinear part of the action we find the propagators

$$
\left\langle\left(H_{\ell}\right)_{j}^{i}\left(V_{\ell}^{\dagger}\right)_{l}^{k}\right\rangle=\left\langle\left(H_{\ell}^{\dagger}\right)_{j}^{i}\left(V_{\ell}\right)_{l}^{k}\right\rangle=\frac{1}{N} \delta_{l}^{i} \delta_{j}^{k} ;\left\langle\left(V_{\ell}^{\dagger}\right)_{j}^{i}\left(V_{\ell}\right)_{l}^{k}\right\rangle=\frac{\tilde{\alpha}_{1}}{N} \delta_{l}^{i} \delta_{j}^{k}
$$

The vertex $\tilde{\beta}_{n} \operatorname{tr} \mathbf{V}_{p}^{n}$ is associated with the loop $(\partial p)^{n}$ going $n$ times around the boundary $\partial p$ of the elementary plaquette $p$. We attache to the loop $(\partial p)^{n}$ a little planar surface $p_{n}$ called $n$-plaquette. The $n$-plaquette $p_{n}$ has area $n$ (we assume that the elementary plaquette has unit area) and a boundary going $n$ times along the boundary of the plaquette $p$. The first three multi-plaquettes $(n=1,2,3)$ are shown in fig. 2 where for eye's convenience we have displaced the edges of the boundary.

The $n$-plaquette with $n>1$ has a singularity (branch point of order $n$ ) containing Gaussian curvature $2 \pi(n-1)$ at some point, say, at its centre. The $n$-plaquettes will serve as building blocks for constructing the world sheet of the lattice string. The Boltzmann weight of an $n$-plaquette in $\tilde{\beta}_{n}$. The propagator $\mathbf{V}-\mathbf{V}$ has simple geometrical meaning: it glues two edges of multiplaquettes having opposite orientations. 
The absence of a $H-H$ propagator means that the $\mathbf{H}$ field just play the role of glue for identifying the edges of the multiplaquettes. Besides the simple contraction $\mathbf{V}-\mathbf{V}$ there are cyclic contractions of $n$ pairs of oppositely oriented edges, $n=2,3$, ., which are represented by the vertices $\tilde{\alpha}_{n} \operatorname{tr}\left(\mathbf{H}^{\dagger} \mathbf{H}\right)^{n}$. The edges are glued half-by-half in a cyclic way. The surface obtained in this way has a singularity at the middle of the link where a curvature $2 \pi(n-1)$ is concentrated. We will call as before this singularity a branch point of order $n$. The Boltzmann factor associated with a cyclic contraction of order $n$ is $\tilde{\alpha}_{n}(\ell)$. The simple contraction and the first nontrivial ones are shown in fig. 3.
$n=1$
$n=2$
$n=3$

Figure 2. Multiplaquettes of orders 1, 2, 3

$$
n=1 \quad n=2 \quad n=3
$$

Figure 3. Cyclic contractions of $n$ edges, $n=1,2,3$

The vacuum Feynman diagrams are surfaces composed of multiplaquettes glued together along their edges by means of cyclic contractions. An important feature of these surfaces is that they can have branch points (that is, local singularities of the curvature) not only at the sites, as it is the case in the original Weingarten model, but also at the links and plaquettes of the surface. These singularities can be interpreted as processes of splitting and joining of strings. The weight of a closed surface is a product of the weights of its elements times a power of $N$ which, with our normalizations, is equal to the Euler characteristics of the surface. 
The free energy of the $U(\infty)$ gauge theory is given by the sum over all closed connected surfaces with the topology of a sphere.

The connection with the traditional Feynman-like diagrams is by duality. Sometimes it is mire convenient to use the traditional diagrammatical notations. Then a surface bounded by a loop $\Gamma=\left[\ell_{1} \ell_{2} \ldots \ell_{n}\right]$ will be represented by a planar Feynman graph with $n$ external legs dual to the links $\ell_{1}, \ldots, \ell_{n}$. The cyclic contraction of $2 n$ is represented by a vertex with $2 n$ lines as is shown in fig. 4 .

Figure 4. Vertices dual to cyclic contractions

Applying these Feynman rules to the Wilson loop average $W(\Gamma)=\left\langle\frac{\operatorname{tr}}{N} \mathbf{V}(\Gamma)\right\rangle$ we find a representation of $W(\Gamma)$ as the sum over all planar surfaces bounded by the contour $\Gamma$. The Boltzmann weight of a surface is the product of the mean values (3.38) associated with the multiplaquettes and cyclic contractions. The local Boltzman weights can be decomposed into factors contributing to the total area of the surface and the length of its boundary, and factors related to the branch points. We denote

$$
\tilde{\beta}_{n}=\tilde{\alpha}_{n}=\tilde{\alpha}_{1}^{n} \omega_{n}^{(1)}, \quad \tilde{\beta}_{1}^{n} \omega_{n}^{(2)}
$$

Then the string path integral for the Wilson loop reads

$$
W(\Gamma)=e^{-m_{0} L[\Gamma]} \sum_{\mathcal{S}: \partial \mathcal{S}=\Gamma} e^{-M_{0} A[\mathcal{S}]} \Omega[\mathcal{S}]
$$

where $L[\Gamma]$ is the length of the contour $\Gamma, A[\mathcal{S}]$ is the area of the surface $\mathcal{S}$, the bare string tension $M_{0}$ and the boundary mass $m_{0}$ are related to the original variables $\tilde{\alpha}_{1}, \tilde{\beta}_{1}$ by

$$
e^{-M_{0}}=\tilde{\alpha}_{1}^{2} \tilde{\beta}_{1}^{\prime} \quad e^{-m_{o}}=\sqrt{\tilde{\alpha}_{1}}
$$

and the $\Omega$-factor is the product of the local factors $\omega_{n}^{(\sigma)}$ associated with the branch points.

The $\Omega$-factor of the surface $\mathcal{S}$ containing $\mathcal{N}_{n}^{(\sigma)} n$-plaquettes, $n=1,2, \ldots$, and $\mathcal{N}_{n}^{(1)}$ cyclic contractions of order $n, n=1,2, \ldots$, is given by

$$
\Omega[\mathcal{S}]=\prod_{n=1}^{\infty} \prod_{\sigma=1,2}\left(\omega_{n}^{(\sigma)}\right)^{\mathcal{N}_{n}^{(\sigma)}}
$$

ant the area of the surface is

$$
A=\sum_{n=1}^{\infty} n \mathcal{N}_{n}^{(2)}
$$




\subsection{Irreducible surfaces (a miracle)}

Unlike the random walk, the two-dimensional surface can exist in configurations with very uneven intrinsic geometry. The typical syngularity is a "neck" representing an intermediate closed string state of small connecting a "baby universe" with the main body of the surface. The most singular configurations with necks can be readily removed from the path integral of the string. Their contribution can be taken into account by modifying the local Boltzmann weights associated with cyclic contractions of edges. Miraculously, the new Boltzmann weights become easily calculable and do not depend neither on the dimension $D$ nor on the choice of the one plaquette action! This is why we will restrict the sum over surfaces to irreducible ones which will render the random surface Ansatz much simpler than it its original version.

The configurations to be excluded are surfaces with necks occupying a single link (fig. 5). We call such a surface reducible with respect to this link..

Figure 5. A reducible surface

In what follows by sum over surfaces we will understand a sum over irreducible surfaces defined as follows.

Definition:

A surface $\mathcal{S}$ is reducible with respect to given link $\ell \in \mathcal{L}$ if it splits into two or more disconnected pieces after being cut along this link. A surface which is not reducible with respect to any $\ell \in \mathcal{L}$ is is called irreducible.

\subsection{Evaluation of the weights of the cyclic contractions}

Consider the Wilson loop average for the contour $\Gamma=\left(\ell_{1} \ell_{2} \ldots\right)$ with coinciding endpoints. 
We will exploit the unitarity condition $\mathbf{V} \mathbf{V}^{\dagger}=\mathbf{I}$, applied to the loop amplitude. It means that the Wilson average $W(\Gamma)$ will not change if a backtracking piece $\ell \ell^{-1}$ is added to the contour $\Gamma$

$$
W\left(\Gamma \ell \ell^{-1}\right)=W(\Gamma)
$$

The sum over surfaces spanning the loop $\Gamma \ell \ell^{-1}$ can be divided into two pieces

$$
W\left(\Gamma \ell \ell^{-1}\right)=W(\Gamma) W\left(\ell \ell^{-1}\right)+W_{\text {conn }}\left(\Gamma \ell \ell^{-1}\right)
$$

The first term is the sum over all surfaces made of two disconnected parts spanning the loops $\Gamma$ and $\ell \ell^{-1}$. The second term contains the rest. The constraint (4.7) is satisfied for all loops if $W\left(\ell \ell^{-1}\right)=1$ and $W_{\text {conn }}\left(\Gamma \ell \ell^{-1}\right)=0$. But there is only one irreducible surface spanning the loop $\ell \ell^{-1}$; it contains a single contraction $\tilde{\alpha}_{1}(\ell)$ between $\ell$ and $\ell^{-1}$. Therefore $\tilde{\alpha}_{1}=1$.

Now consider a surface contributing to the second term $W_{I}$. The links $\ell$ and $\ell^{-1}$ may be connected to the rest of the surface by means of the same contraction or by two cyclic contractions The condition that their total contribution is zero is

$$
\tilde{\alpha}_{n}+\sum_{k=1}^{n-1} \tilde{\alpha}_{k} \tilde{\alpha}_{n-k}=0, \quad n=2,3, \ldots
$$

This equation is illustrated in fig. 6 by means of the standard graphical notations (fig. 4). Eq. (4.9) is identical to the loop equation satisfied by the coefficients $f_{n}$ in the expansion (3.23), which is solved by the Catalan numbers

$$
\tilde{\alpha}_{n}=f_{[n]}=(-)^{(n-1)} \frac{(2 n-2) !}{n !(n-1) !}
$$

Figure. 6. Graphical representation of the unitarity condition

The second derivation is based only on the the sum over surfaces for the trivial Wilson loops

$$
W\left[\left(\ell \ell^{-1}\right)^{n}\right]=\left\langle\frac{\operatorname{tr}}{N}\left(\mathbf{V}_{\ell} \mathbf{V}_{\ell}^{\dagger}\right)^{n}\right\rangle=1, n=1,2, \ldots
$$

For each of these Wilson loops the sum over irreducible surfaces contains only finite number of terms, namely, the link-vertices contracting directly the edges of the loop $\ell \ell^{-1}$. For example,

$$
W\left(\ell \ell^{-1}\right)=\tilde{\alpha}_{1}, W\left[\left(\ell \ell^{-1}\right)^{2}\right]=2 \tilde{\alpha}_{1}^{2}+\tilde{\alpha}_{2}, \ldots
$$


Introducing the generating functions

$$
w(t)=\sum_{n=0}^{\infty} t^{n} W\left[\left(\ell \ell^{-1}\right)^{n}\right]=\frac{1}{1-t}, \quad f(t)=1+\sum_{n=1}^{\infty} t^{n}\left(\tilde{\alpha}_{n}\right)^{n}
$$

we easily find the relation [1]

$$
w(t)=f\left[t w^{2}(t)\right]
$$

which is solved by the function generating the Catalan numbers

$$
f(t)=\frac{1+\sqrt{1+4 t}}{2}
$$

In this way, unlike the $U(N)$ vector model, the classical values of the auxiliary fields do not depend on the dimension of the space-time. The expectation value $\tilde{\alpha}$ in the vector model is determined by the long wave excitations of the random walk. Here in the gauge theory, the expectation values of the fields $\tilde{\alpha}_{n}$ are determined in purely local way, as it is clear from their derivation. One way to explain this difference is the local character of the $U(N)$ invariance in the case of the gauge theory.

On the contrary, the weights of the multiplaquettes will depend on the dimension as well as on the choice of the one-plaquette action. If wee are interested only in the large $N$ limit, it is more convenient not to try to calculate them by solving the equations of motion but just to take them as independent coupling constants. The continuum limit (if it exists) then will be achieved along a trajectory

$$
\tilde{\beta}_{n}=\tilde{\beta}_{n}(\lambda, D), \quad \lambda \rightarrow 0
$$

where $\lambda$ is the coupling constant.

\subsection{String representation of the Wilson loop average. Resumé}

Let us summarize what we have achieved by now. The Wilson loop average $W(\Gamma)$ in the $U(\infty)$ gauge theory defined on a $D$-dimensional lattice is equal to the sum of all planar irreducible surfaces having as a boundary the loop $\Gamma$. These surfaces are allowed to have branch points of all orders located at the sites, links and plaquettes of the space-time lattice. Introducing unified notations $\omega_{n}^{(k)}$ for the weights of the branch points associated with the $k$-cells of the space-time lattice (sites are 0-cells, links ate 1-cells, and plaquettes are 2-cells) where

$$
\begin{aligned}
& \omega_{n}^{(0)}=1 \\
& \omega_{n}^{(1)}=f_{[n]}=(-)^{(n-1)} \frac{(2 n-2) !}{n !(n-1) !} \\
& \omega_{n}^{(2)}=\frac{\tilde{\beta}_{n}}{\left(\tilde{\beta}_{1}\right)^{n}}
\end{aligned}
$$

we can write the sum over surfaces as

$$
W(\Gamma)=\sum_{\partial \mathcal{S}=\Gamma} \Omega(\mathcal{S}) e^{-M_{0} \operatorname{Area}(\mathcal{S})}
$$

where the factor $\Omega(\mathcal{S})$ is a product of the weights of all branched points of the world sheet and $M_{0}=-\ln \tilde{\beta}_{1}$. 


\subsection{The contribution of the surfaces with folds is zero}

The weights of the branch points provide a mechanism of suppressing the backtracking motion of the strings or, which is the same, world surfaces having folds. The surfaces with folds have both positive and negative weights and their total contribution to the string path integral is zero. We have checked that in many particular cases but the general proof is missing.

It is perhaps instructive to give one example. Consider the surface in fig. 7 .

Figure 7. A piece of surface having a fold

It covers three times the interiour of the nonselfintersecting loop $C$ and once - the rest of the lattice. There are two specific points on the loop $C$ at which the curvature has a conical singularity; they can be thought of as the points where the fold is created and annihilated. Each of these points can occur either at a site or at a link (in the last case it is associated with a weight factor $\omega_{2}^{(1)}=f_{[2]}=-1$ ).

Let us evaluate the total contribution of all irreducible surfaces distinguished by the positions of the two singular points. Denoting by $n_{0}$ and $n_{1}\left(=n_{0}\right)$ the number of sites and links along the loop $C$, and remembering that the a singular point located at a link has to be taken with a weight $f_{[2]}=-1$, we find that the contribution of these surfaces is proportional to

$$
\left[\frac{n_{0}\left(n_{0}-1\right)}{2}+f_{[2]} n_{0} n_{1}+f_{[2]}^{2} \frac{n_{1}\left(n_{1}-1\right)}{2}\right]-\left[n_{1}+2 f_{[2]} n_{1}\right]=0
$$

The second term on the left hand side contains the contribution of the reducible surfaces which had to be subtracted. A reducible surface arises when the two points are located at the extremities of the same link ( $n_{1}$ configurations) or when one of the points is a branch point and the other is located at one of the extremities of the same link $\left(2 n_{1}\right.$ configurations). 


\subsection{Loop equations}

In ref. [11] we proved that the loop equations in the Wilson lattice gauge theory [20] are satisfied by the the sum over surfaces (4.18). Let us only write here the general formula which is derived in the same fashion. For any link $\ell \in \Gamma$

$$
\sum_{n=1}^{\infty} \sum_{p: \partial p \ni \ell} \tilde{\beta}_{n}\left[\left(W\left(\Gamma(\partial p)^{n}\right)-W\left(\Gamma(\partial p)^{-n}\right)\right]=\sum_{\ell^{\prime} \in \Gamma} W\left(\Gamma_{\ell \ell^{\prime}}\right) W\left(\Gamma_{\ell^{\prime} \ell}\right)\left[\delta\left(\ell, \ell^{\prime}\right)-\delta\left(\ell^{-1}, \ell^{\prime}\right)\right]\right.
$$

The sum on the l.h.s. goes over the $2(D-1)$ plaquettes adjacent with the link $\ell$ and the closed loops $\Gamma_{\ell \ell^{\prime}}, \Gamma_{\ell^{\prime} \ell}$ in the contact term are obtained by cutting the links $\ell$ and $\ell^{\prime}$ and reconnecting them in the other possible way.

\subsection{The trivial $D=2$ gauge theory as a nontrivial model of random surfaces}

The case $D=2$ was considered recently in details in [21]. For the heat kernel action the string tension $M_{0}$ and the weights $\omega_{n}^{(2)}$ are given by

$$
M_{0}=\frac{\lambda}{2} ; \quad \omega_{n}^{(2)}=\sum_{m=1}^{n-1}\left(\begin{array}{c}
n \\
m+1
\end{array}\right) \frac{n^{m-1}}{m !}(-\lambda)^{m}=\left(1-\frac{n(n-1)}{2} \lambda+\ldots\right)
$$

Let us illustrate how the string Ansatz (4.18) works for the simplest nontrivial example of a Wilson loop (fig. 8) which have been calculated previously using the Migdal-Makeenko loop equations [22].

Figure 8. A contour with the form of a flower and a branched surface bounded by it

The nonfolding branched surfaces spanning the loop will cover the three petals of the flower (denoted by 1 in fig. 8 ) once, and the head (denoted by 2 ) - twice. Therefore the total area will be always $A=A_{1}+2 A_{2}$, where $A_{i}$ is the area enclosed by the domain $i(i=1,2)$. Each of these surfaces will have a branch point located at some site, link or cell of the overlapping area 2 . 
Let us denote by $n_{0}, n_{1}, n_{2}$ the numbers of sites, links, cells belonging to the domain 2 . Then the sum over the $\Omega$-factors due to the branch points reads

$$
\sum \Omega=\sum_{k=0,1,2} n_{k} \omega_{2}^{(k)}=\left(n_{0}-n_{1}+(1-\lambda) n_{2}\right)=1-n_{2} \lambda
$$

(Here we used the Euler relation $n_{0}-n_{1}+n_{2}=1$.) Therefore

$$
W(C)=\left(1-A_{2}\right) e^{-\frac{1}{2}\left(A_{1}+2 A_{2}\right)}
$$

\section{Strong versus weak coupling}

Let us first discuss the case of the Wilson action where the mean field problem is solved exactly in the large $N$ limit [17]. It is well known that when $N \geq 4$ and $D=4$, the strong coupling phase of a theory with Wilson action is separated from the continuum limit by a nondeconfining first-order phase transition. The assumption that $\mathbf{H}_{\mathrm{cl}}=\mathbf{V}_{\mathrm{cl}}=0$ is a local minimum of the free energy is justified in the strong coupling phase of the gauge theory. In the weak coupling phase the matrix fields will develop vacuum expectation values. The classical fields form an orbit of the gauge group

$$
\mathbf{V}_{<x y>}=\mathbf{U}_{x} \mathbf{V}_{\mathrm{cl}} \mathbf{U}_{y}^{-1}, \quad \mathbf{H}_{<x y>}=\mathbf{U}_{x} \mathbf{H}_{\mathrm{cl}} \mathbf{U}_{y}^{-1}
$$

The diagram technique of sect. 2 then has to be modified according to the general rules explained in [12]. The surface elements will contain free edges, and, as a consequence, windows will appear spontaneously on the world sheet of the string. Whether these windows destroy or not the world sheet in the continuum limit is a dynamical question. Believing in the confinement, we expect that in $D \leq 4$ dimensions the windows are still not sufficiently large for that, and the world sheet will have in the continuum limit the structure of a dense network of thin strips separating the windows (fig. 1). These strips will correspond to the gluon propagators in the standard Feynman rules. At the critical dimension $D=4$ the effective string tension of the string (with windows on the world sheet) should scale with the coupling $\lambda$ according to the asymptotic freedom law, just as the mass in the vector model does in $D=2$ dimensions.

In $D>4$ dimensions the world sheet of the string is eaten by one or several large windows and the Wilson loop behaves as exponential of the length of the contour.

Whether this picture is true in geleral, can be decided by studying the mean field problem for a general one-plaquette action. 


\section{References}

[1] K. Wilson, Phys. Rev. D 10 (1974) 2445; A. M. Polyakov, unpublished

[2] G. 't Hooft, Nucl. Phys. B72 (1974) 461

[3] I. Bars, Journ. Math. Phys. 21 (1980) 2678; S. Samuel, Journ. Math. Phys. 21 (1980) 2695; R.C. Brower and M. Nauenberg, Nucl. Phys. B180 (1981) 221; D. Weingarten, Phys. Lett. 90B (1980) 280; D. Foerster, Nucl. Phys. B170 (1980) 107

[4] B. Rusakov, Mod. Phys. Lett. A5 (1990) 693

[5] M. Douglas and V. Kazakov, preprint LPTENS-93/20

[6] V.Kazakov, Phys. Lett. 128B (1983) 316, JETP (Russian edition) 85 (1983) 1887

[7] H.E. Stanley, Phys. Rev. 176 (1968) 718

[8] D. Weingarten, Phys. Lett. 90B (1980)285

[9] K.H. O'Brien and J.-B. Zuber, Nucl. Phys. B253 (1985) 621, Phys. Lett. 144B (1984) 407

[10] I. Kostov, Phys. Lett. 138B (1984) 191, 147B (1984) 445

[11] I. Kostov, Nucl. Phys. B265 (1986) 223

[12] J.-M. Drouffe and J.-B. Zuber, Phys. Rep. 102, Nos. 1,2 (1983)1-119, section 4

[13] D. Gross and E. Witten, Phys. Rev. D21 (1980) 446

[14] J.M. Drouffe and C. Itzykson, Statistical Field Theory, Cambridge University Press 1989

[15] A. M. Polyakov, Phys. Lett. 72B (1978) 447; J.-M. Drouffe, Phys. Rev. D18 (1978) 1174; L. Susskind, Phys. Rev. D20 (1979) 2610; M. Nauenberg and D. Toussaint, Nucl. Phys. B190 (1981) 288; P. Menotti and E. Onofri, Nucl. Phys. B190 (1984) 288; C.B. Lang, P. Salomonson and B.S. Skagerstam, Phys. Lett. 107B (1981) 211, Nucl. Phys. B190 (1981) 337

[16] P. Rossi, Ann. Phys. 132 (1981) 463

[17] E. Brézin and D. Gross, Phys. Lett. 97B (1980) 120

[18] K.H. O'Brien and J.B. Zuber, Phys. Lett. 144 B (1984) 407

[19] M. Douglas, Preprint RU-93-13 (NSF-ITP-93-39); J. Minahan and A. Polychronakos, hep-th/9303153

[20] A.A. Migdal, unpublished (1978); D. Förster, Phys. Lett. 87B (1979) 87; T. Eguchi, Phys. Lett. 87B (1979) 91

[21] I. Kostov, Saclay preprint T93/050, 1993; submitted to Nucl. Phys. B

[22] V.Kazakov and I. Kostov, Nucl. Phys. B176 (1980) 199, V. Kazakov, Nucl. Phys. B179 (1981) 283 